\title{
Focused low intensity shock wave therapy for management of benign prostatic hyperplasia: A Preliminary study
}

\author{
A.A.Abou-Taleb, W.S.Kandeel, A.A. El-Shaer and K.M.Noah \\ Urology, Dept., Faculty of Medicine, Benha Univ., Benha, Egypt \\ E-Mail:Kareemnoah1088@gmail.com
}

\begin{abstract}
Objective: assess the effectiveness of shock waves in bph management by patients who do not react to medical treatment Method and patients: This research is a prospective, non-randomized single-arm study at Benha University Hospital's Urology Department. This comprised (32) individuals with BPH, with or without at least 6 months of poor response to pharmacological treatment by one or more $\alpha$-blockers, poor surgical candidates for co-morbidities or not interested in surgery. All patients underwent six LISWT sessions once a week, and all patients were assessed by changes in IPSS, QMAX, IIEF5, and PVRU at 3rd and 6th, and 3 months after treatment completion Results: substantial improvement was seen in the maximal flow rate from $9 \pm 1.50 \mathrm{ml} / \mathrm{sec}$ before to sessions beginning to $13.9 \pm 1.2 \mathrm{ml} / \mathrm{sec}$, and also in PVR from $79.34 \pm 56.87 \mathrm{ml}$ to $55.43 \pm 35.65 \mathrm{ml}$ during the sixth week of follow-up. The IPSS also improved substantially from $28 \pm 4.5$ to $20.34 \pm 5$ at the 6 th week of follow-up. Conclusion:LISWT is safe to use for BPH treatment particularly in non-respondent medical therapy without severe side effects based on its antispasmodic characteristics.
\end{abstract}

Keywords: Scockwaves, prostate, LUTS, lowintenisty, BPH.

\section{Introduction}

Benign prostatic hyperplasia (BPH) is one of the most prevalent illnesses among ageing men that may result in decreased symptoms of the urinary tract (LUTS). The incidence of BPH increases significantly with age. Autopsy investigations found a histological prevalence of $8 \%, 50 \%$ and $80 \%$ respectively throughout the fourth, sixth and ninth decades of life [1].

Multiple methods of therapy for BPH have been developed, including lifestyle changes, $\alpha$-blockers $(\alpha 1$ - adrenoceptors), $5 \alpha$-reductase inhibitors, phytochemicals and BPH associated surgery [2].

Surgical methods and less invasive treatments include various degrees of side effects and consequences including ejaculatory dysfunction, ED, urethral restrictions, and urine incontinence (2-3). Exploring a stronger and more safe BPH approach is thus a major issue for doctors, and physical therapy is increasingly focused on functional urology.

The first effective use of extracorporeal shock wave treatment (ESWT) to lithotripsis in urology. At lower energy levels, however, shock waves increased production of vascular endothelial growth factor, endothelial synthase of nitric oxides, proliferating nuclear cells, chemoattractor factors and progenitor cell recruitment [4].

ESWT was described as helpful in muscles, including hypertonia of the muscle, capsular and calcific tendinitis through neovascularization processes, anti-inflammatory mechanisms and regeneration of the tissue [5]

For the last decade, fESWT was found as virtually non-negative in the perineal approach, which has significantly improved symptoms of chronic pelvic pain syndrome, vagueness (6-7-8-9) and a penis approach for ED treatment (10-11-12).
2. Patients and method.

\subsection{Study design and patients}

This study is prospective, nonrandomized,single-arm study that conducted at Urology Department,Benha university hospital .It included (32) patients with BPH who are poorly responding to medical therapy with one of the $\alpha$ blockers with or without finasteride for at least 6 months, poor candidates for surgical intervention due to comorbidities, or not interested in surgery.all the patients received 6 sessions of LISWT once aweek and all the patients were evaluated at $3^{\text {rd }}$ session and $6^{\text {th }}$ session and 3 months after the end of the caurse by change in IPSS, QMAX, IIEF5 and PVRU Urinary tract stone. Patients with urethral stricture, urinary tract infection ,primary renal disease, neurogenic bladder, history of prostate cancer, bladder cancer, previous prostate or urethral surgery were excluded, the patients were evaluated using IPSS ,QMAX, IIEF and PVRU at $3^{\text {rd }}$ and $6^{\text {th }}$ session and after 3 months

\subsection{Treatment schedule}

The device used is The Piezowave 2 of Richard Wolf GmbH and All participants received 6 sessions of LISWT once a week .Each time 5000 pulses of $0.16 \mathrm{~mJ} / \mathrm{mm} 2$ with rate $6 \mathrm{~Hz}$

\subsection{Follow up and assessment}

The patients were evaluated using IPSS ,QMAX, IIEF and PVRU at $3^{\text {rd }}$ and $6^{\text {th }}$ session and after 3 months

\subsection{Statistical analysis}

The collected data were tabulated and analyzed using SPSS version 21 software (Spss Inc, Chicago, ILL Company).quantitative data were expressed as mean \pm standard deviation, and range. Repeated measure ANOVA and pearson's correlation coefficient ( $r$ ) were used as tests of significance. Significant 
ANOVA was followed by Bonferroni test .The accepted level of significance in this work was stated at 0.05 ( $\mathrm{P}<0.05$ was considered significant).

Table (1) descriptive data for participants

\begin{tabular}{lcccc}
\hline & Mean & S.D & Minimum & Maximum \\
\hline age & 55.3 & 3.333 & 45 & 54 \\
IPSS & 28.34 & 4.2 & 19 & 35 \\
Q max & 9.7 & 1.4 & 3 & 14 \\
PVR & 60.78 & 34.78 & 30 & 300 \\
\hline
\end{tabular}

Table (2) follow up parameters at $3^{\text {th }}$ week

\begin{tabular}{lcccc}
\hline & Mean & S.D & minimum & maximum \\
\hline IPSS at 3 week & 26.3 & 4.2 & 20 & 30 \\
Q max at 3weeks & 10.3 & 1.5 & 5 & 20 \\
PVR at 3weeks & 55.44 & 49.23 & 23 & 190 \\
IIEF at 3weeks & 10,45 & 2.67 & 0 & 26 \\
\hline
\end{tabular}

At $3^{\text {th }}$ week there was improvement in the IPSS from $26.4 \pm 4.2$ before starting sessions to $18.45 \pm 3.4$.

There was also significant increase in QMAX and IIEF from $5.7 \pm 3.5$ and 10.56 \pm 2.5 at time of the presentation to $6.4 \pm 2.9$ and $8.4 \pm 3.78$ at the $3^{\text {th }}$ session respectively .

Table (3) follow up parameters at $6^{\text {th }}$ week

\begin{tabular}{lcccc}
\hline & Mean & S.D & minimum & Maximum \\
\hline IPSS at 8 week & 26.3 & 4.2 & 20 & 30 \\
Q max at 8 weeks & 10.3 & 1.5 & 5 & 20 \\
PVR at 8 weeks & 55.44 & 49.23 & 23 & 190 \\
IIEF at 8 weeks & 10,45 & 2.67 & 0 & 26 \\
\hline
\end{tabular}

At $6^{\text {th }}$ week there was improvement in the IPSS from $26.4 \pm 4.2$ before starting sessions to $18.45 \pm 3.4$.There was also significant increase in QMAX and IIEF from $5.7 \pm 3.5$ and $10.56 \pm 2.5$ at time of the presentation to $6.4 \pm 2.9$ and $8.4 \pm 3.78$ at the $3^{\text {th }}$ session respectively .

Table (4) follow up parameters at $3^{\text {rd }}$ month

\begin{tabular}{lcccc}
\hline & Mean & S.D & Minimum & Maximum \\
\hline IPSS at 6 months & 26.3 & 4.2 & 20 & 30 \\
Q max at 6 months & 10.3 & 1.5 & 5 & 20 \\
PVR at 6 months & 55.44 & 49.23 & 23 & 190 \\
IIEF at 6 month & 10,45 & 2.67 & 0 & 26 \\
\hline
\end{tabular}

At $3^{\text {rd }}$ month there was improvement in the IPSS from $26.4 \pm 4.2$ before starting sessions to $18.45 \pm 3.4$

There was also significant increase in QMAX and IIEF from $5.7 \pm 3.5$ and $10.56 \pm 2.5$ at time of the presentation to $6.4 \pm 2.9$ and $8.4 \pm 3.78$ at the $3^{\text {th }}$ session respectively .

\section{Discussion}

In ageing men, the growth of the prostate gland is caused by gradual hyperplasia in the stream and glands, smooth muscle and epithelial cells, and the spread of collagen fibrils and calcification inside the transitional prostate area. Smooth muscle spasm is another possible mechanism of LUTS induced by BPH [14]

However, the adverse effects of such drugs vary greatly, including tiredness, dizziness, headache, and postural hypotension, which have established safety and effectiveness, so it may be very unpleasant to take these medicines in the elderly, particularly those treated with additional antihypertensive drugs [14].

Medical therapy with a $5 \alpha$-reductase inhibitor needs a longer treatment time to achieve maximum impact [14].

The effects of low-intensity shock wave treatment on relieving LUTS caused by BPH are based on muscle tone decreasing and hypertonia, which have been proposed to contribute as a potential LUTS mechanism. The increased muscle tone of the prostatic tissue smooth musculoskeletal fibres may make the patient symptoms caused by BPH worse [8]. 
Muscle tone and spasticity are reduced after ESWT application for patients with upper arm hypertonia and hypertonic plantar flexor due to a stroke [8].

Results from our research revealed that six ESWT perineal sessions may lead to substantial improvements of the IPSS from $26.4 \pm 4.2$ before sessions began to $18.45 \pm 3.4$.

QMAX and IIEF also increased significantly from $5.7 \pm 3.5$ and $10.56 \pm 2.5$ at the presentation time to $6.4 \pm 2.9$ and to $8.4 \pm 3.78$ respectively at the 3 rd session.

Although the data appears very promising, there are several limitations which must be taken into account: (a) the follow-up period of only 3 months is short; the durability of this approach is not known and long-term data is waiting for with interest and (b) the number of cases is small and no control group is limited. This technique, despite the unknown mechanism of action and the aforementioned constraints, may actually constitute substantial progress. Basic research is also necessary to understand the mechanism for therapy Finally, the noninvasive rESWT physical therapy promises to be a LUTS treatment in relation to BPH. This technique is possibly feasible in a wide variety of drug-refractory patients who may or may not be interested in surgery. This research may guide the design and execution of a randomised, sham-controlled pivotal test.

\section{Conclusion}

Schok waves can be used safely in management of LUTS related to $\mathrm{BPH}$.

\section{References}

[1] S.Gravas, JN.Cornu, MJ. Drake, M.Gacci, C.Gratzke and TRW.Herrmann, Management of non-neurogenic male lower urinary tract symptoms (LUTS).vol.5,99-180,2018.

[2] M.Stephan, S.Natalie and C.Zoran: Pathophysiology of Benign Prostatic Hyperplasia and Benign Prostatic Enlargement: A Mini-ReviewGerontology.vol.65,pp.458464,2019.

[3] H.Masuda, T.Okuno, M.Suzuki, K.Kihara, M.Goto and H.Azuma: Different distribution of nitric oxide synthase and soluble guanylyl cyclase activities in the detrusor and proximal urethra of the rabbit.vol.90, pp.168-286,2002.

[4] W.Bloch, T.Klotz, C.Loch, G.Schmidt, U.Engelmann and K.Addicks: Distribution of nitric oxide synthase implies a regulation of circulation, smooth muscle tone, and secretory function in the human prostate by nitric oxide. Prostate.vol.33,pp.1-8,1997.

[5] R.Zimmermann, A.Cumpanas, F.Miclea and G.Janetschek. Extracorporeal shock wave therapy for the treatment of chronic pelvic pain syndrome in males: a randomised, double-blind, placebo-controlled urology.vol.56(3),pp.418-424,2009.
[6] I.Gruenwald, B.Appel, N. D. Kitrey, and Y. Vardi.Shockwave treatment of erectile dysfunction. Therapeutic Advances in Urology.vol.5(2),pp.95-99,2013.

[7] Y.Vardi, B.Appel, A.Kilchevsky, and I. Gruenwald, Does low intensity extracorporeal shock wave therapy have a physiological effect on erectile function? Short term results of a randomized, double-blind, sham controlled study. The Journal of Urology.vol.187(5),pp.1769-1775,2012.

[8] G.Gotte, E.Amelio and S.Russo. Short-time non-enzymatic nitric oxide synthesis from Larginine and hydrogen peroxide induced by shock waves treatment. FEBS letters.vol.520(13),pp.153-155,2002.

[9] K.Hatanaka, K.Ito, T.Shindo, Y.Kagaya, T.Ogata, K.Eguchi, and H.Shimokawa. Molecular mechanisms of the angiogenic effects of low-energy shock wave therapy: Roles of mechanotransduction. American Journal of Physiology. Cell Physiology.vol.311(3),pp.378-385,2016.

[10] R.Assaly-Kaddoum, F.Giuliano, M.Laurin, D.Gorny, M.Kergoat, J.Bernabe, and D.BehrRoussel.Low intensity extracorporeal shock wave therapy improves erectile function in a model of type II diabetes independently of NO/cGMP pathway. The Journal of Urology.vol.196(3),pp.950-956,2016.

[11] T. H.Huang, C. K.Sun, Y. L.Chen, C. J.Wang,T. C.Yin, M. S. Lee, and H. K. Yip.Shock wave enhances angiogenesis through VEGFR2 activation and recycling. Molecular Medicine.vol.22(1),pp.1-150,2016.

[12]Dai Zhang , Yun-Lei Wang , Da-Xin Gong , Zhao-Xuan Zhang, Xiao-Tong Yu, and YueWen Ma. American Journal of Men's Health January-February .vol.55,pp.50-56,2019.

[13] Jennifer Westwood, Robert Geraghty, Patrick Jones, P.Bhavan Rai and K.Bhaskar Somani. Rezum: a new transurethral water vapour therapy for benign prostatic hyperplasia Ther Adv Urol.vol.7,pp.59-90,2018.

[14] Hamidi Madani, A.Enshaei, A.Heidarzadeh, M G.okhtari, A.Farzan, Mohiti Asli, M.and S.Esmaeili, Transurethral intraprostatic botulinum toxin-A injection: A novel treatment for BPH refractory to current medical therapy in poor surgical candidates. World Journal of Urology.vol.31(1),pp.235-239,2013. 